\section{Chinese herbal decoction - Xuming Tang: a possible effective treatment for ischemic stroke patients}

\section{Abstract}

So far, rt - PA remains the best treatment for ischemic stroke within the 3-6 hour window after the onset. However, the majority of ischemic stroke patients do not receive $r t$ - PA treatment due to various reasons and they can only accept the treatment which prevents a second attack. The Chinese herbal decoction - Xuming Tang has been recorded as an effective treatment for stroke in the traditional Chinese medicine book. Recently it has been tested in over 1,500 patients who started taking this herbal decoction from 2.5 hours to days after the onset of stroke in randomised and quasi-randomised trials. It has been shown that approximately $1 / 3$ of the stroke patients fully recovered and another $1 / 3$ improved dramatically after the treatment period. Compared with the data from the control group which received either conventional therapy (this prevents a second attack) or conventional therapy in combination with other Chinese herbal decoctions, Xuming Tang is a better choice for ischemic stroke patients who are ineligible for $r t$ - PA thrombolysis within 3-6 hour window or who miss this time window.
Huazheng Liang ${ }^{1,2}$, Shaoshi Wang ${ }^{1}$

1 Department of Neurology, Sichuan Branch of No.1 People's Hospital, Jiaotong University

2 Neuroscience Research Australia

Corresponding author:

Huazheng Liang

”. h.liang@neura.edu.au

\section{Facts about Ischemic stroke and its treatment}

Ischemic stroke is the most common type of stroke worldwide. Each year over 2 million patients are diagnosed with this disease and the cost of medical care for these patients has been dramatically increasing in China'.
So far, rt - PA remains the most effective treatment for ischemic stroke within 3 hours onset ${ }^{2}$. There are clinical trials extending the time window for using rt - PA to 4.5 hours $^{3-6}$ or 6 hours ${ }^{7-9}$. Certain groups of ischemic stroke patients are likely to benefit from this. However, the fact is that less than $20 \%$ of patients can receive $r t$ - PA due to the delay in visiting a hospital or being excluded from receiving $r t$ - PA 
thrombolysis ${ }^{10-15}$. Therefore, the majority of ischemic stroke patients are treated with conventional medications for secondary prevention. Though a considerable proportion of ischemic stroke patients will recover with these medications and the subsequent rehabilitation, most of these patients will be left with either disability or apparent symptoms. This poses a large burden for the patients, carers, and the health care system.

\section{Xuming Tang for ischemic stroke}

Xuming Tang is a Chinese herbal decoction for stroke treatment recorded in "Xiaopin Fang" by a Chinese medicine doctor Yanzhi Chen living in the period of 420AC-589AC ${ }^{16}$. This herbal decoction has been refined by other Chinese medicine doctors after him. The main ingredients include ephedra, semen armeniacae amarum, rambles cinnamomi, rhizome chuanxiong, radix angelica sinensis, radix glycyrrhizae, dry ginger, gypsum fibrosum, radix codonopsis. These are the same for all patients. Other herbs are also included based on the condition of the patients. For example, patients with too much "Yang" will receive radix scutellariae, radix trichosanthis, rhizoma anemarrhenae, and patients with too much "Cold" will receive aconitum carmichaeli debx, herba asari, pericarpium zanthoxyli. According to the Chinese medicine theory, this herbal decoction activates the circulation to resolve blood stasis, dispels interior and exterior "Wind", tonifies "Qi" and blood, clears extra "Heat", disperses "Qi", removes "Dampness", and induces diuresis to alleviate edema. As a result, the upward and downward transportation of nutrients absorbed in the "Stomach" and "Spleen" is restored and resuscitation is achieved ${ }^{17}$. From the point view of western medicine, this decoction protects the brain tissue by stopping the influx of extracellular calcium and minimizing the permeability of capillaries ${ }^{18,19}$, prevents further injury caused by NO, oxidized lipids ${ }^{20}$, and calcium; lowers the blood pressure in hypertensive patients and the level of lipids including cholesterol and triglycerol ${ }^{20,21}$, inhibits the aggregation of platelets and the formation of thrombus, prolongs the time of coagulation, and improves the blood supply to the brain ${ }^{19}$.

Recently, many Chinese medicine doctors began to test its efficacy for stroke, especially ischemic stroke, through randomized or quasi-randomised trials (see table 1). They recruited patients with ischemic stroke arriving at the hospital 2.5 hours to over 5 days after the onset. Both male and female patients at any age were included. These patients were divided into two groups. The treatment group received Xuming Tang decoction and the control group received either placebo or conventional medications for secondary prevention, or conventional medications with other Chinese herbal recipes for ischemic stroke. The treatment period varied from 8 days to 8 months with a majority between 2 weeks and 6 weeks. The result from meta-analysis of these trials showed that approximately one third of the patients in the treatment group fully recovered without any symptoms or signs of neurological deficits, whereas only $23 \%$ of the patients in the control groups fully recovered. Apart form this, slightly over one third of the patients in the treatment group had apparent improvement demonstrated by a reduction of $45-90 \%$ of neurological deficit scale and a 2 grades increase in muscle strength. In comparison, only about a quarter of patients in the control group had the same improvement.

These results show that Xuming Tang decoction is an effective therapy for ischemic stroke patients who miss the 3-6 hour window for rt - PA thrombolysis. It not only reduces the chance of getting disabled but also cures some of the patients as rt - PA does in timely treated patients. Therefore, it reduces the burden of the patients, carers, and the 
Table 1. Data from published articles

\begin{tabular}{|c|c|c|c|c|c|c|c|c|c|c|c|}
\hline \multicolumn{7}{|c|}{ Xuming Tang } & \multicolumn{5}{|c|}{ control } \\
\hline Authors & year & group 1 & group 2 & group 3 & group 4 & total & group 1 & group 2 & group 3 & group 4 & total \\
\hline Gu & 1989 & 118 & 56 & 17 & 9 & $200 *$ & & & & & \\
\hline Huang & 1989 & & 46 & 41 & 1 & $88^{*}$ & & & & & \\
\hline $\mathrm{Hu}$ & 1995 & 26 & 52 & 19 & 1 & $98^{*}$ & & & & & \\
\hline Yu & 1996 & 20 & 6 & 4 & 0 & 30 & 12 & 4 & 6 & 6 & 28 \\
\hline Chen & 1997 & 10 & 11 & 9 & 1 & 31 & 3 & 7 & 7 & 3 & 20 \\
\hline Yu & 1998 & 22 & 24 & 12 & 2 & 60 & 6 & 10 & 9 & 5 & 30 \\
\hline Zhan & 1999 & 8 & 16 & 6 & 2 & 32 & 5 & 9 & 11 & 3 & 28 \\
\hline Zhang & 1999 & 42 & 36 & 19 & 15 & $112^{*}$ & & & & & \\
\hline LV & 2006 & 12 & 15 & 7 & 6 & 40 & 4 & 10 & 6 & 18 & 38 \\
\hline $\mathrm{Hu}$ & 2010 & 15 & 16 & 9 & 5 & 45 & 8 & 8 & 14 & 11 & 41 \\
\hline Tian & 2010 & & 14 & 20 & 1 & 35 & & 11 & 17 & 3 & 31 \\
\hline Zhang & 2011 & 5 & 14 & 8 & 3 & 30 & 4 & 9 & 9 & 8 & 30 \\
\hline Zhou & 2011 & 9 & 23 & 6 & 2 & 40 & 5 & 22 & 11 & 2 & 40 \\
\hline Liu & 2012 & 21 & 10 & 5 & 2 & 38 & 16 & 9 & 7 & 5 & 37 \\
\hline LiG & 2012 & & 16 & 29 & 5 & 50 & & 11 & 26 & 13 & 50 \\
\hline LiJ & 2012 & 65 & & 28 & 8 & 101 & 42 & & 34 & 21 & 97 \\
\hline Li & 2013 & 15 & 11 & & 2 & $28^{*}$ & & & & & \\
\hline Gong & 2013 & 7 & 13 & 8 & 2 & 30 & 3 & 8 & 4 & 5 & 30 \\
\hline Total & & 395 & 379 & 247 & 67 & 1088 & 108 & 118 & 171 & 103 & 500 \\
\hline$n \%$ & & $35.8(33.6)$ & $36.0(34.3)$ & $22.3(25.4)$ & $5.9(6.4)$ & 100 & 23.1 & 24.5 & 32.2 & 20.3 & 100 \\
\hline
\end{tabular}

group 1: cured or with no neurological deficits

group 2: dramatic improvement by 2 grades increase in muscle strength

group 3: improvement by 1 grade increase in muscle strength

group 4: no changes or get worse

*indicates no control groups

$\mathrm{N}(\mathrm{n})$ : $\mathrm{N}$ indicates percentage by including the trials without control groups, (n) indicates percentage by excluding the trials without control groups. 
health system. It brings hope to those patients with ischemic stroke and their treatment with rt -PA is delayed or excluded due to various reasons.

\section{Questions and the future of Xuming Tang}

Undoubtedly, the above results will be questioned concerning the method used in these clinical trials. Firstly, the time range of arriving at the hospital after the onset of ischemic stroke has a large variation. This time difference will influence the randomization and the subsequent analysis of the result. Secondly, each patient is receiving different combinations of herbs. This poses a question about the efficacy of the herbs which differ between patients. Thirdly, the treatment period varies dramatically between patients, which affects the evaluation of neurological deficits, muscle strength, and disability.

Among these trials, some of the investigators analyzed the efficacy of Xuming Tang on different types of stroke based on the diagnosis by Chinese medicine doctors. They found that Xuming Tang is more effective for patients with "Wind" and "Phlegm" stasis, or "Dampness-Phlegm syndrome" than those with any other forms of stroke ${ }^{22}$. This indicates that multiple mechanisms are responsible for stroke development. Therefore, treatment for each stroke patient should be tailored.

Some investigators studied the effect of Xuming Tang on intracerebral hemorrhage and found that patients with intracerebral hemorrhage also benefit from this decoction compared with the conventional therapy ${ }^{23}$. This highlights the commonality shared by the ischemic and hemorrhagic stroke in disease etiology, development, and treatment.

These challenges require further better designed, large scaled trials to verify the proficiency of Xuming
Tang on ischemic stroke patients who are treated 6 hours after the onset. If this is confirmed, it might be necessary to compare the efficacy of Xuming Tang with that of rt - PA in ischemic stroke patients who are treated within 3 or 6 hours after the onset. After all, this is a hope for ischemic stroke patients who are either ineligible for rt - PA thrombolysis within the 3-6 hour window or who miss the time window for rt - PA thrombolysis. Furthermore, more research is encouraged to investigate the cause, mechanism, and therapeutics of both the ischemic and hemorrhagic stroke.

\section{References}

1. Liu, L., Wang, D., Wong, K.S., Wang, Y. Stroke. 42(12), 3651-4 (2011).

2. NINDS rt-PA Stroke Study Group. N. Engl. J. Med. 333, 15811587 (1995).

3. Hacke, W. et al. J Am Med Assoc. 274, 1017-1025 (1995).

4. Hacke, W. et al. Lancet. 352, 1245-1251 (1998).

5. Steiner, T. et al. Cerebrovasc. Dis. 8, 198-203 (1998).

6. Hacke, W. et al. N. Engl. J. Med. 359, 1317-1329 (2008).

7. Hacke, W. et al. Lancet. 363, 768-774 (2004).

8. Lees, K. et al. Lancet 375, 1695- 1703 (2010).

9. Whiteley, W., Lindley, R., Wardlaw, J., Sandercock, P. Int. J. Stroke. 1, 172-6 (2006).

10. Qureshi, A. et al. Neurosurgery. 57, 647-654 (2005).

11. Katzan, I. et al. J. Am. Med. Assoc. 283, 1151-1158 (2000).

12. Katzan, I. et al. Arch. Neurol. 61, 346-350 (2004).

13. Katzan, I., Hammer, M., Furlan, A., Hixson, E., Nadzam, D. Stroke. 34, 799-800 (2003).

14. Johnston, S. et al. Stroke. 32, 1061-1068 (2001).

15. Hills, N. \& Johnston, S. Am. J. Prev. Med. 31, S210-S216 (2006).

16. Chen, Y. Xiaopin Fang. Nanbei Chao Dynasty. In Gao, W. Xiaopin Fang (edited). 1995, Chin Press T. C. M. Beijing.

17. Pan, H.X. J. Zhejiang T. C. M 12 (5), 521-522 (2001).

18. Ye, Y.Y. et al. Chin. J. Exp. T. C. M. 5(3), 48-49 (1999).

19. Chen, L.F., Wang, X.H., Peng, Z.H. Pharmacol. Clin. Chin. Materia. Medica. 13(6), 6-8 (1997).

20. Wang, J.P. \& Tang, N. Shenzhen. J. Integrated. Traditional. Chinese. Western. Med. 1(19), 13- 15 (2009).

21. Zheng, J.H., Wang, S.M., Yang, W.D. et al. Pharmacol. Clin. Chin. Materia. Medica. 3(10), 13-14(1996).

22. Chen, L.F., Liu, H.X., Ye, M. Hunan. J. T. C. M. 13(6), 5-6 (1997).

23. Hu, M.L. Yunnan. J. T. C. M. Materia. Medica. 31(2), 34-35 (2010). 


\section{Comment on this article:}

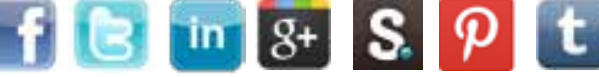

Where Doctors exchange clinical experiences,
review their cases and share clinical knowledge.
You can also access lots of medical publications for
free. Join Now!

\section{Publish with iMedPub}

\section{http://www.imed.pub}

Neurology and Neuroscience (jneuros.com) is a hybrid, peerreviewed journal that considers articles concerned with any aspect of clinical neurosciences such as neurology, psychiatry and neurosurgery, as well as basic research on neuroscience where neurologists and neuroscientists publish together.

Submit your manuscript here: http://www.jneuros.com 